\title{
Design and Simulation Analysis of a 120 MPa Deep-Sea Ultra-High Pressure Environment Simulation Test Chamber
}

\author{
Kaiben YU,a,b, Shengqi YU ${ }^{\mathrm{a}, \mathrm{b}, 1}$, Qingjian MENG ${ }^{\mathrm{a}}$, Le ZONG ${ }^{\mathrm{a}}$ and Baohua Liu ${ }^{\mathrm{a}, \mathrm{b}}$ \\ a National Deep Sea Center, Ministry of Natural Resources, China \\ ${ }^{\mathrm{b}}$ Laboratory for Marine Geology, Qingdao National Laboratory for Marine Science \\ and Technology, China
}

\begin{abstract}
According to the requirements of pressure resistance and sealing performance detection of deep-sea instruments and equipment under full sea pressure, a 120MPa deep-sea test chamber for ultra-high pressure environment simulation is designed in this paper. The structural dimensions of the test chamber were designed and calculated, including the cabin wall thickness, the cabin bottom head thickness, the sealing top cover, and the locking nut. The strength of the cylinder structure and the sealing cover of the test chamber were verified and analyzed through finite element analysis, which validates the reliability of the test chamber structure. The $120 \mathrm{MPa}$ deep-sea test chamber for ultra-high pressure environment simulation designed in this paper can provide important technical support for testing the pressure resistance and the sealing performance of deep-sea instruments and equipment.
\end{abstract}

Keywords. Full sea pressure, Environment simulation, Design calculation, Simulation analysis.

\section{Introduction}

Deep-sea areas contain rich important strategic resources indispensable for national economic development, and have become a field highly focused on by various countries in the world in recent years. Deep submersibles operating in various deep-sea environments have become the most effective tools for exploration, investigation and exploitation of submarine resources [1]. In the development and design process of deep submersibles and various deep-sea instruments and equipment, pressure test of parts is an essential link. Direct implementation of deep-sea experiment relates to high cost, great difficulty and high risk, and is easily affected by such external factors as environmental conditions, so it is hard to ensure the experimental accuracy [2]. In recent years, with the constant increase in the field and depth of deep-sea scientific investigation, scientific research on environmental, geological and biological aspects in hadal zones represented by the Mariana Trench (water depth of $\sim 11,000 \mathrm{~m}$ ) in the Pacific Ocean has become a new hot spot. Therefore, a kind of deep-sea environment simulation device that can simulate the pressure environment at water depth of 12,000

\footnotetext{
${ }^{1}$ Corresponding Author, Shengqi YU, National Deep Sea Center, Ministry of Natural Resources, China; Laboratory for Marine Geology, Qingdao National Laboratory for Marine Science and Technology, China; Email: ysq@ndsc.org.cn.
} 
$m$ is urgently needed, to provide support to pressure environment simulation test for the technical equipment for deep-sea investigation in the Mariana Trench.

In this paper, a $120 \mathrm{MPa}$ ultra-high pressure test chamber system was designed based on the current test requirements for deep-sea instruments and equipment during development, and it enabled automatic control of pressure rise and decline, to effectively simulate ten thousand meter level deep-sea environments.

In this paper, the structural design of the $120 \mathrm{MPa}$ deep-sea ultra-high pressure test chamber cabin was mainly carried out, and simulation analysis verification was conducted with ANSYS for the strength of cabin structure. The results show that the design parameters of this test chamber fully meet the technical requirements and it can provide pressure and sealing performance test for deep-sea instruments and equipment.

\section{Technical Requirements for and Composition of the 120 Mpa Deep-Sea Ultra- High Pressure Environment Simulation Test Chamber}

\subsection{Technical Requirements for Pressure and Dimensions of the $120 \mathrm{MPa}$ Ultra-high} Pressure Test Device

Based on the current requirements for and technical situations of test of deep-sea instruments and equipment, the test pressure for the deep-sea ultra-high pressure test chamber was set to $120 \mathrm{MPa}$ and the effective internal dimensions of the chamber were set to $500 \mathrm{~mm}$ of internal diameter and $1,500 \mathrm{~mm}$ of effective length.

\subsection{Composition of the 120 MPa Ultra-high Pressure Test Chamber}

With the use situation of the ultra-high pressure test chamber being taken into account, the ultra-high pressure test chamber was designed as a form of a cylinder plus a large U-shaped structure on the upper, to utilize the effective test space in the cylinder to the maximum extent [1][3]. This static pressure test chamber employed a structure of cabin forging and welding by section, bracket O-ring sealing and nut locking according to the Chinese specifications for design of ultra high pressure vessels [4] by referencing the structures of the 18 inch (internal diameter) static pressure test chamber (rated pressure: 20,000 psi) at the Pennsylvania State University, USA (as shown in figure 1) and the $440 \mathrm{~mm}$ (internal diameter) static pressure test chamber (rated pressure: $100 \mathrm{MPa}$ ) in the Center of Oceanographic Standards and Metrology of State Oceanic Administration, etc. [5][6] It was mainly composed of such parts as cabin, sealing bracket, sealing top cover, locking nut, locking arm, inner-hexagon column-head screw, hanging ring screw for locking nut, hanging ring screw for top cover, and O-ring seal. The specific composition is shown in figure 2 . 


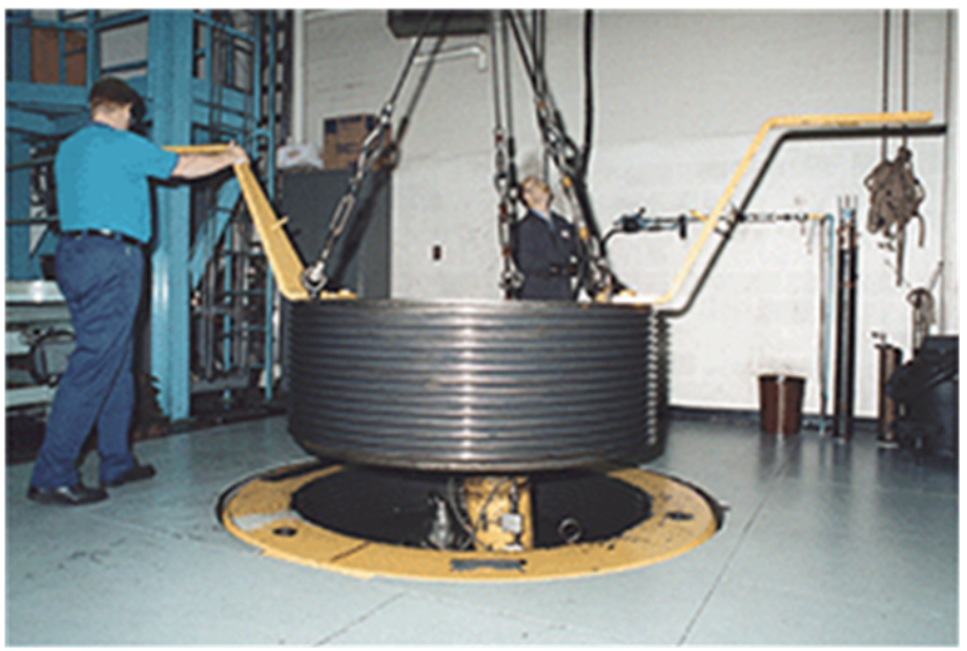

Figure 1. Static pressure test chamber at the Pennsylvania State University.

\section{Structural Design of the 120 MPa Ultra-high Pressure Test Chamber}

\subsection{Design of Cabin Dimension Parameters}

The cabin was made of $20 \mathrm{MnMoNb}$ alloy structural steel through forging and welding by section, and the tensile strength of the material was $o_{b} \geq 635 \mathrm{MPa}$.

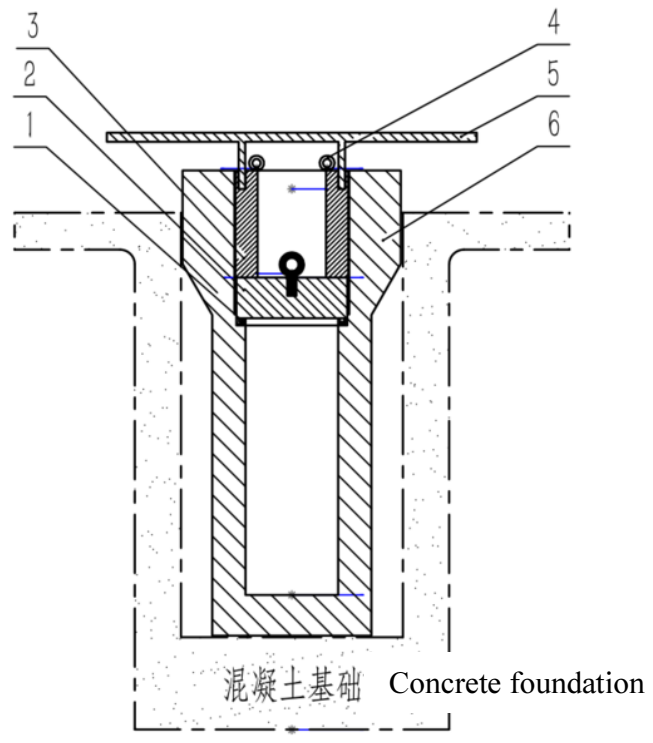

Figure 2. Structural diagram of ultra-high pressure test chamber. 1-Sealing bracket, 2-Sealing top cover, 3Locking nut,4-Hanging ring screw for locking nut, 5-Locking arm, 6-Cabin. 


\subsubsection{Design of Cabin Wall Thickness}

According to design formula for cylinder wall thickness, the cabin wall thickness could be calculated with the following equation:

$$
\delta=P D /(2 \sigma \phi-P)
$$

where $\delta$ is the cylinder wall thickness $(\mathrm{mm}), P$ is the design pressure (MPa), $D$ is the internal diameter of cabin $(\mathrm{mm}), \sigma$ is the allowable stress of material $(\mathrm{MPa})$, and $\varnothing$ is the weld joint factor.

The design parameters were substituted into the equation, to obtain the calculation result of the cabin wall thickness as $\delta=120 \times 500 /(2 \times 254 \times 0.9-120)=178 \mathrm{~mm}$.

Based on the calculation result, the actual design cabin wall thickness was $180 \mathrm{~mm}$.

\subsubsection{Design of Cabin Bottom Head Thickness}

The cabin bottom head thickness was calculated with the following equation:

$$
h=0.433 D\left(5 P / \sigma_{b^{-}}\right)^{-2}
$$

where $h$ is the head thickness (mm), and $\sigma_{b}$ - is the tensile strength of material (MPa). The design parameters, including safety factor of 5 , were substituted into the equation, to obtain the calculation result of bottom head as $h=0.433 \times 500 \times(5 \times 120 / 635)^{-2}=$ $210 \mathrm{~mm}$. Based on the calculation result, the cabin bottom head was designed as 220 $\mathrm{mm}$.

\subsubsection{Design of Cabin Locking Threads}

Since the cabin locking nuts bore only unidirectional load, it was planned to use GB/T13576.3-1992 serrated thread. The locking thread had a nominal diameter of 600 $\mathrm{mm}$ and a pitch of $12 \mathrm{~mm}$. The material of the locking but had a tensile strength of $\geq 635 \mathrm{MPa}$ and a yield strength of $\geq 490 \mathrm{MPa}$. The shear stress of the locking thread should satisfy the following equation:

$$
\tau=\frac{F}{\pi D b z} \leq[\tau]
$$

where the axial force $F=P A=26460780 \mathrm{~N}$, the major diameter of thread $D=600 \mathrm{~mm}$, the thread tooth bottom width $b=0.736 P=0.736 \times 12=8.832 \mathrm{~mm}$, the number of engaged turns $z=32$. $[\tau]$ is the allowable shear stress in MPa, taken as $[\tau]=0.6[\sigma]$. $[\sigma]$ is the allowable tensile stress of material, $\sigma=\sigma_{s} / S$, where $\sigma_{s}$ is the yield stress in MPa and the safety factor is taken as $S=5$.

The design parameters were substituted into the equation, to obtain the calculation result of shear stress of locking thread as $\tau=26460780 /(3.14 \times 600 \times 8.832 \times 32)=$ $49.695<[\tau]=0.6 \times 490 / 5=58.8 \mathrm{MPa}$.

As can be discerned from the calculation, the thread strength meets the requirements and the thread is safe.

\subsection{Dimension Design of the Sealing Top Cover}

The cabin top cover was forged with $35 \mathrm{CrMo}$ alloy structural steel, and the tensile strength of the material was $\geq 980 \mathrm{MPa}$. The top cover thickness design was the same as 
the calculation equation for the cabin bottom head thickness. The cabin design parameters were substituted into the equation, to obtain the calculated top cover thickness as $h=0.433 \times 530 \times(5 \times 120 / 980)^{-2}=180 \mathrm{~mm}$.

The actual top cover thickness was designed as $220 \mathrm{~mm}$ based on the calculation result, with such strength attenuation factors as top cover opening being taken into account.

\subsection{Design of the Locking Nut}

Corresponding to the cabin locking thread, the locking nuts were forged with 35CrMo alloy structural steel, the tensile strength of the material was $\geq 980 \mathrm{MPa}$, and the maximum calculated load pressure was $214 \mathrm{MPa}$. The GB/T13576.3-1992 serrated thread was used, and its nominal diameter was $600 \mathrm{~mm}$ and its pitch was $12 \mathrm{~mm}$. The strength of this material was higher than that of cabin material, so the strength was no longer checked.

\section{Finite Element Strength Check Analysis of the 120 MPa Ultra-high Pressure Test Chamber Cabin}

\subsection{Finite Element Simulation Modeling of the Cabin}

As the test chamber cabin was designed as an axisymmetric structure, finite element discretization of cabin should be conducted with axisymmetric plane elements in finite element analysis and calculation. A simulation model of cabin was constructed according to the above design of the cabin and parts and the symmetric structure of the test chamber cabin. To analyze the force status of the cabin main structure, the structures of such parts as locking arm, inner-hexagon column-head screw, hanging ring screw for locking nut, hanging ring screw for top cover, and watertight connecting part, were simplified in the modeling.

Firstly, the cabin model was gridded into quadrilateral grid structure, and the gridding effect is shown in figure 3. In addition, to give consideration to both simulation efficiency and simulation precision, the grids in contact zones were fined and those in non-contact zones were enlarged. 


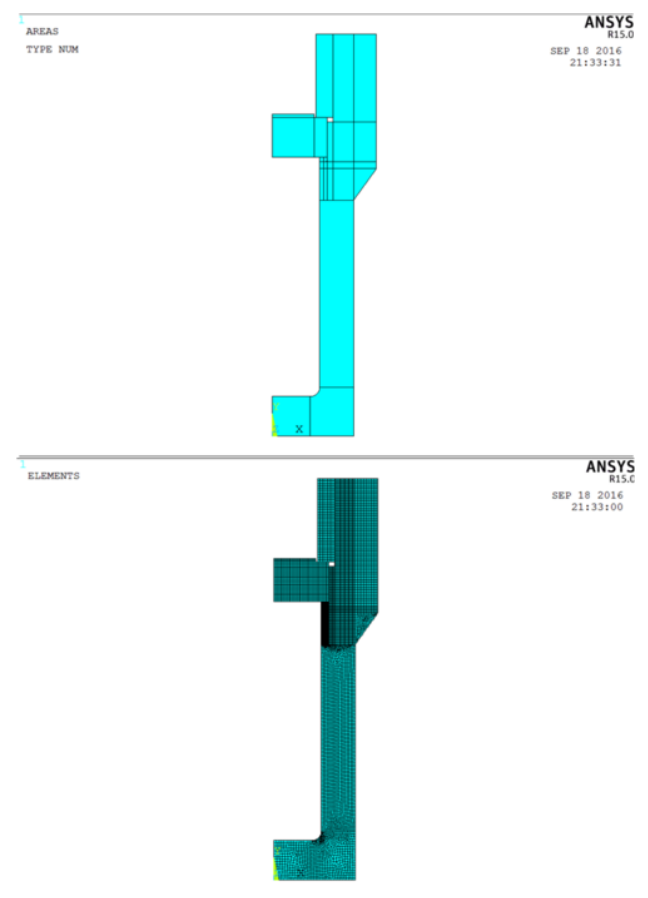

Figure 3. Cross-section model and gridding of cabin.

To reveal the contact stress situations between cabin and locking nut, between cabin and sealing top cover, between cabin and sealing ring, between locking nut and sealing top cover, and between locking nut and sealing ring, it was planned to use the contact elements (contact172 and target169) in the ANSYS to construct a model, and the steel-steel friction coefficient was taken as 0.15 . Since the locking nut and cabin were in fixed state during actual mounting, their contact relationship in the ANSYS simulation process was selected as Bonded(always). Furthermore, the calculation was carried out using the algorithm of "Augmented Lagrange method" [7].

\subsection{Setting of Simulation Boundary Conditions}

Based on the deformation feature of axisymmetric structure, the following boundary constraints were applied to the finite element calculation model of pressure cabin:

(1) Symmetric constraint condition was applied to the central line nodes;

(2) To eliminate the displacement of rigid body, axial displacement constraint condition was applied to the nodes on the symmetric line of locking nut;

(3) As can be discerned from the fact that the pressure cylinder was mounted in semiburied environment, the upper external surface and bottom of the cabin were fixed with concrete structure, so fixed constraint was applied to the cabin bottom and axial constraint was applied to the upper external surface of the cabin in the analysis.

(4) The non-linear states between cabin, sealing top cover, sealing ring and locking nut were simulated with linear contact elements. 


\subsection{Simulation Analysis}

The design pressure of ultra-high pressure test chamber was $120 \mathrm{MPa}$. This pressure was taken as the calculation pressure in the strength analysis and it directly acted on the elements of cabin inner wall, sealing ring and sealing top cover bottom.

\subsubsection{Analysis of Deformation of Pressure Chamber Cabin Structure}

Figure 4 shows the diagrams of deformation of pressure cabin structure. It can be seen from the figure that, owing to the effect of the test pressure, the cylindrical cabin has some outward volume expansion, with maximum radial displacement reaching 3.208 $\mathrm{mm}$. The maximum axial deformation of the cabin occurs at the center of the sealing top cover. The top cover undergoes water pressure and the surrounding locking nuts constrains the upward free movement of the top cover edge so that the displacement at the center of the top cover is the most evident, reaching $1.55 \mathrm{~mm}$.

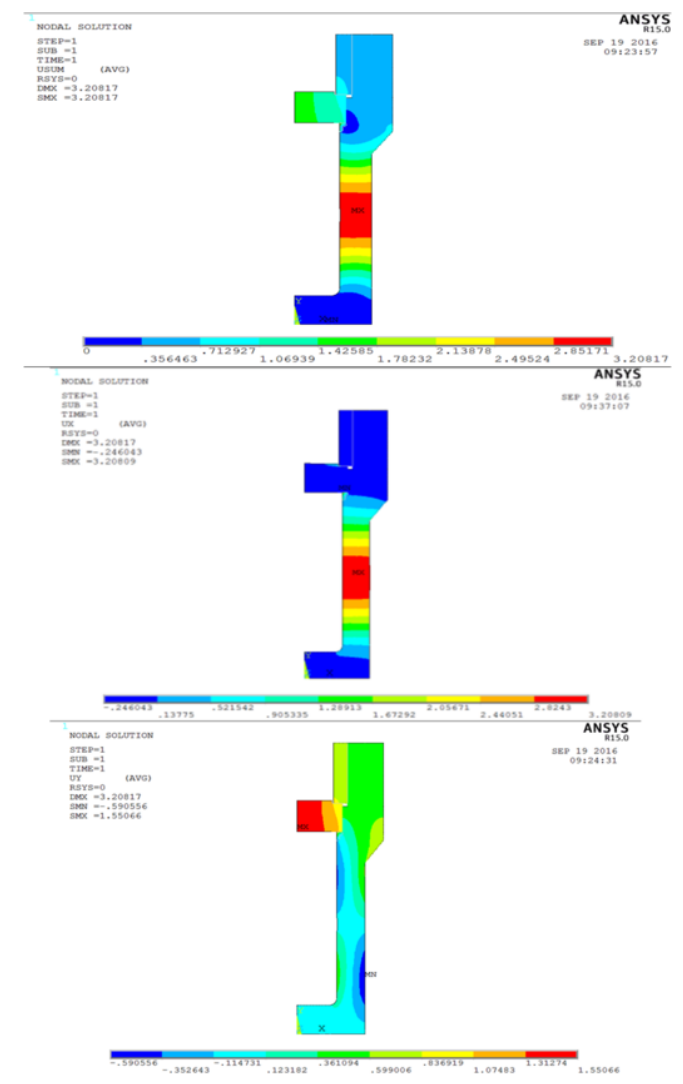

(a)

(b)

(c)

Figure 4. Simulation results of deformation of cabin structure.

(a) Overall deformation. (b) Radial deformation. (c) Axial deformation.

\subsubsection{Analysis of Deformation of Pressure Cabin Top Cover Structure}

Figure 5 shows the diagrams of deformation of pressure cabin sealing top cover structure. As can be discerned from the figure, the top cover, under the constraint effects of the test pressure and locking nuts, is in an upward bending state, with the upper surface in tension and the lower surface in compression, and with maximum deformation of $1.55 \mathrm{~mm}$. Undergoing the squeezing by shear, the peripheral margin of 
upper surface of the top cover is the place, with minimum axial displacement, of the top cover.

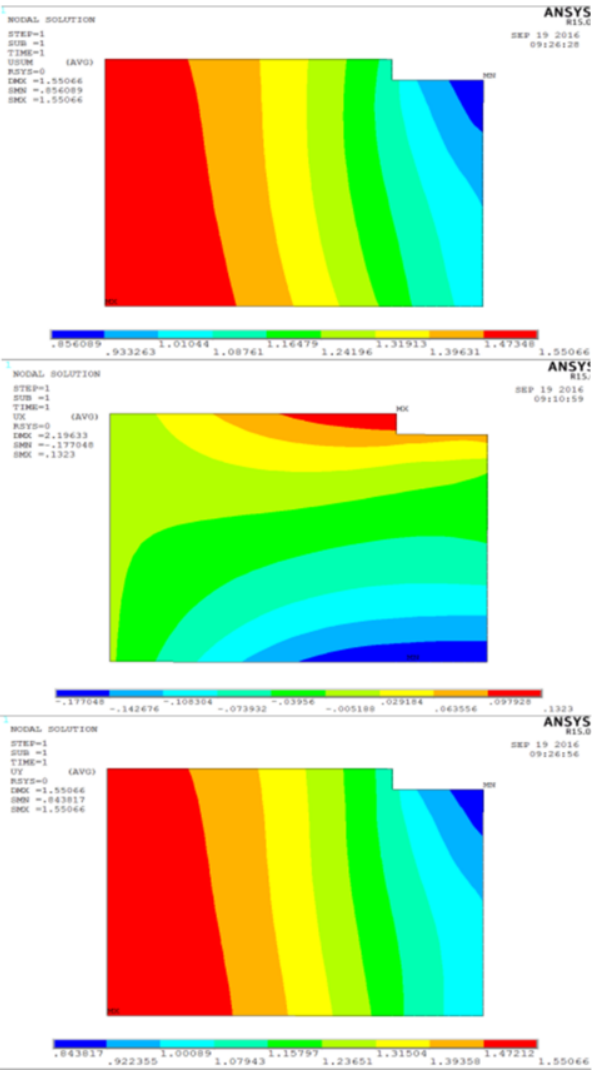

(a)

(b)

(c)

Figure 5. Simulation results of deformation of sealing top cover structure. (a) Overall deformation. (b) Radial deformation. (c) Axial deformation.

\subsubsection{Analysis of Stress of Pressure Chamber Cabin and Sealing Top Cover}

Figure 6 shows the diagrams of stress distribution of pressure chamber cabin and sealing top cover. According to the radial and axial contour diagrams, the stresses at the bottom fillet transition place of cabin are relatively large, and the radial and axial stresses are $667.384 \mathrm{MPa}$, and 839.251 MPa, respectively, both of which exceed the yield limit of the material, so local plastic deformation occurs. This is stress concentration caused by structural discontinuity. The stress concentration at local spots cannot represent the force status of the whole structure, so the cabin and the sealing top cover were separately analyzed additionally.

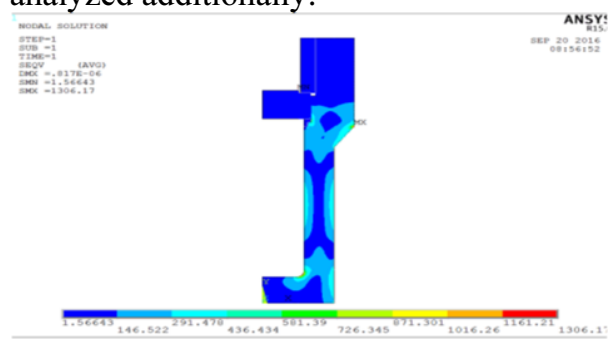




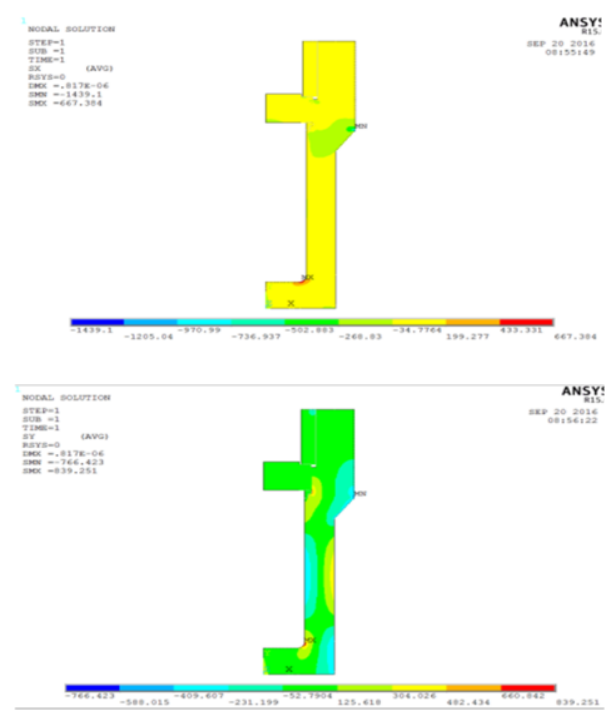

(b)

(c)

Figure 6. Contour diagram of stress. (a) Overall stress. (b) Radial stress. (c) Axial stress.

Figure 7 and figure 8 show the diagrams and distribution laws of stress of the sealing top cover and cabin of the test chamber. As can be discerned from the above figures, the maximum stress of the top cover is $233.98 \mathrm{MPa}$, less than the yield limit of the material ( $835 \mathrm{MPa}$ ), and that of the cabin is $417.86 \mathrm{MPa}$, less than the yield limit of the material (460 MPa). Therefore, in respect of the overall design of the cabin, the structural design of the pressure cabin meets the requirements under the pressure of 120 MPa.
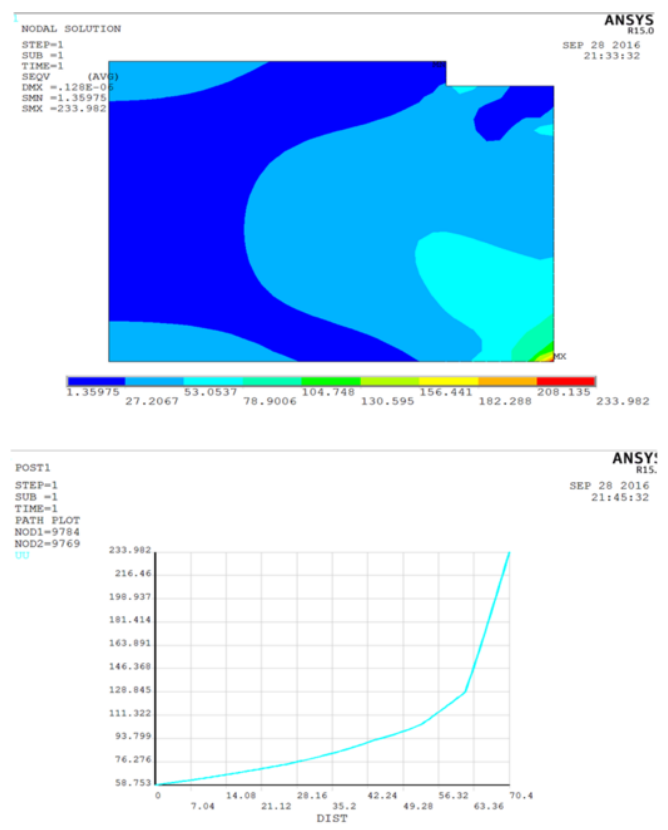

Figure 7. Contour diagram of stress of sealing top cover and simulation results of radial stress. 


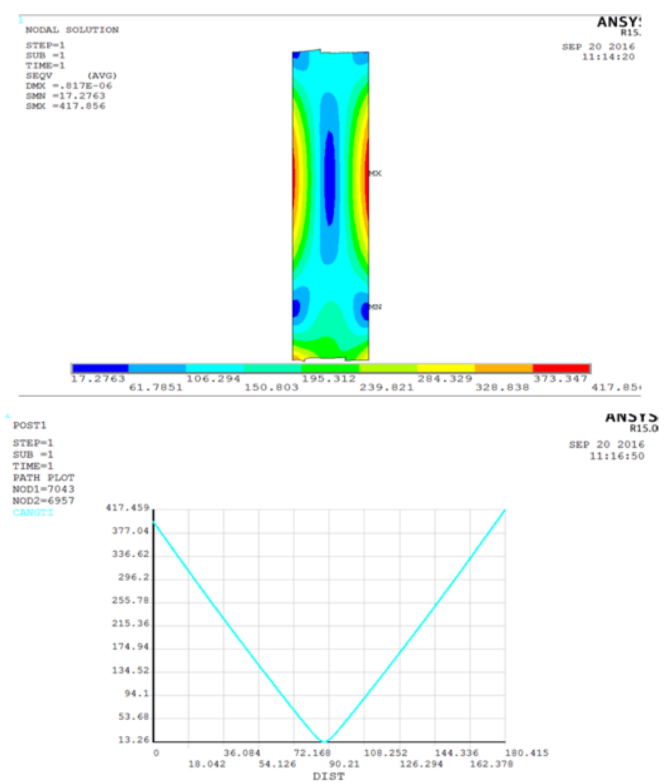

Figure 8. Contour diagram of stress of cabin and simulation results of radial stress distribution.

\section{Conclusions}

In this paper, structural design was carried out for various functional parts of a 120 $\mathrm{MPa}$ deep-sea ultra-high pressure test chamber, and design of parameters was conducted for various parts of the pressure cylinder. Simulation verification of the overall design was carried out with the ANSYS software, demonstrating the rationality of the design. Subsequently, according to the design in this paper, the first large-scale $120 \mathrm{MPa}$ deep-sea ultra-high pressure environment simulation test chamber (figure 9) in China was completed in the National Deep-Sea Base Management Center. It has provided numerous pressure tests for the parts of such deep-sea equipment as Jiaolong and has supported the implementation of such state-level projects as projects of National Natural Science Foundation of China, and key R\&D projects of the Ministry of Science and Technology of PRC. It greatly improves the R\&D capability of China in the aspect of deep-sea technical equipment and promotes the development of deep-sea technology in China. 


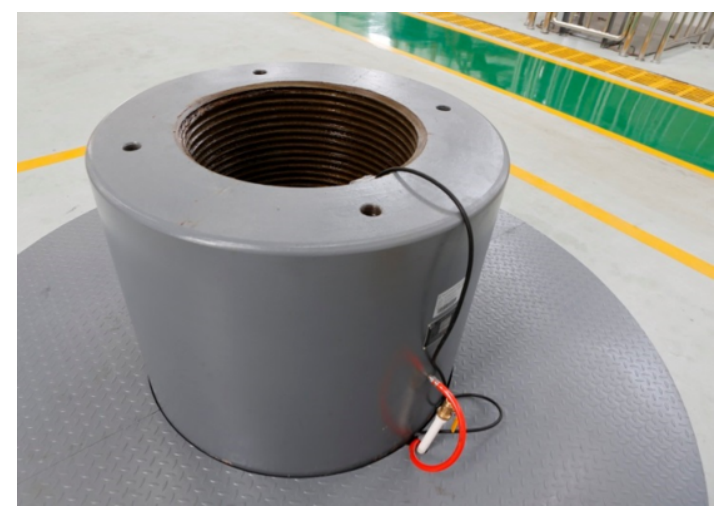

Figure 9. $120 \mathrm{MPa}$ deep-sea ultra-high pressure environment simulation test chamber.

\section{Acknowledgments}

This work was supported by the Shandong Engineering Technology Research Center for Deep-sea Carrier Equipment, Qingdao Public Research and Development Platform for Deep-sea Technology and Equipment, and the Taishan Scholar Project Funding (Grant No. tspd20161007). The authors would also like to express gratitude to the editors and the anonymous reviewers for their constructive comments and suggestions.

\section{References}

[1] Shen Y C, Wan Z Q and Cai X G 2010 Proc. of the Academic Conference 2010 and the Third Meeting of the Sixth Council of Sub-association for Marine and Offshore Steel Structures, CSCS. Jiangsu: Editorial Department of Ship Mechanics, China Ship Science Research Center, p 334

[2] Lu D Q 2009 Structural Design and Research on Deep-sea Environment Simulator (Chengdu: Xi'an Jiaotong University Press)

[3] Wu S W, Li G J and Song X C 2008 Pressure Vessel Technol. 2535

[4] Shao G H 2002 Ultra-high Vessels (Beijing: Chemical Industry Press)

[5] Pan G S and Bian R G 2012 Sci-tech report of China Ship Science Research Center

[6] Pan G S 2013 Sci-tech report of China Ship Science Research Center

[7] Pu G Y 2010 Basic Tutorial and Detailed Examples of ANSYS Workbench (Beijing: China Water \& Power Press) 